\title{
Real-Time Airborne Gamma-Ray Background Estimation Using NASVD with MLE and Radiation Transport for Calibration
}

\author{
J. A. Kulisek, J. E. Schweppe, S. C. Stave, B. E. Bernacki, D. V. Jordan, T. N. Stewart, C. E. \\ Seifert, W. J. Kernan
}

Pacific Northwest National Laboratory, Richland, Washington, 99352, United States

\section{Abstract}

Helicopter-mounted gamma-ray detectors can provide law enforcement officials the means to quickly and accurately detect, identify, and locate radiological threats over a wide geographical area. The ability to accurately distinguish radiological threat-generated gamma-ray signatures from background gamma radiation in real time is essential in order to realize this potential. This problem is non-trivial, especially in urban environments for which the background may change very rapidly during flight. This exacerbates the challenge of estimating background due to the poor counting statistics inherent in real-time airborne gamma-ray spectroscopy measurements.

To address this challenge, we have developed a new technique for real-time estimation of background gamma radiation from aerial measurements without the need for human analyst intervention. The method can be calibrated using radiation transport simulations along with data from previous flights over areas for which the isotopic composition need not be known.

Over the examined measured and simulated data sets, the method generated accurate background estimates even in the presence of a strong, ${ }^{60} \mathrm{Co}$ source. The potential to track large and abrupt changes in background spectral shape and magnitude was demonstrated. The method can be implemented fairly easily in most modern computing languages and environments. 
1 Keywords: background estimation, aerial search, radiological search, gamma detection, noise-

2 adjusted singular value decomposition, maximum likelihood estimation

\section{$3 \quad 1.0$ Introduction}

4 Helicopter-mounted gamma-ray detectors can provide law enforcement officials the means to

5 quickly and accurately detect, identify, and locate radiological threats over a wide geographical

6 area. The ability to accurately distinguish radiological threat-generated gamma-ray signatures

7 from benign, background gamma radiation in real time is essential in order to realize this

8 potential. This problem is non-trivial, especially in urban environments for which the

9 background may change very rapidly during flight over, for example, land-water interfaces and

10 building materials containing large amounts of naturally occurring radioactive material (NORM).

11 This exacerbates the challenge of estimating background due to the poor counting statistics from

12 long standoff distances and short measurement times inherent in real-time airborne gamma-ray

13 spectroscopy measurements.

14 To address this challenge, we have developed a new technique for real-time estimation of

15 background gamma radiation from aerial measurements to support current efforts to significantly

16 enhance the speed, accuracy, and precision of radiological threat detection, measurement, and

17 localization over wide geographical areas. Background estimations from the current algorithm

18 will be used as input, to provide a baseline for anomaly detection [1] and localization [2]

19 algorithms.

20 A prodigious amount of work has been published regarding background estimation techniques,

21 for which a summary is provided in recent literature [3]; although, it was mentioned that most of

22 these techniques require human analyst intervention and were developed for high-count spectra, 
1 high-resolution spectra, and/or spectra containing known sources. More recently, a background

2 estimation technique based on machine learning was developed to estimate the background

3 component in such low-count spectra containing unknown sources, without the need for human

4 intervention [3].

5 The technique described in this paper has also been developed to estimate the background for

6 low-count and low-resolution spectra containing unknown sources without the need for human

7 analyst intervention. The technique described herein is fairly simple and straight-forward to

8 implement using mathematical functions (e.g., singular value decomposition) that are available

9 in pre-existing libraries for several modern computing languages and environments. In addition,

10 we demonstrate that the sensitivity matrix, which is used for extracting the concentrations of the

11 major contributing background components, can be determined through the use of radiation

12 transport simulations. Traditionally, for airborne gamma-ray detection systems, this sensitivity

13 matrix is determined using empirical measurements over pads consisting of known material

14 composition [4].

15 The current algorithm uses data in the high-energy regions or channels of the data to estimate the

16 background signature in the lower channels. This same fundamental concept has been applied in

17 car-borne and airborne search of radioactive materials to determine the background contribution

18 to low-energy spectral windows, using a least-squares approach [5-6]. In this work, we apply

19 Poisson regression, using the method of maximum likelihood estimation (MLE) to estimate the

20 background counts in each channel of the spectrum. Poisson regression naturally incorporates

21 the Poisson statistical nature of the background spectra. To date, the current algorithm has only

22 been analyzed on data taken from a previous flight, in replay mode, to simulate real-time

23 background estimation. 
1 We define gamma-ray background radiation as natural radiation emitted from soil (terrestrial),

2 nearby building materials (e.g., concrete, asphalt, brick, aircraft itself), gamma rays from cosmic-

3 ray showers, and atmospheric radon. The background estimation algorithm described herein

4 assumes that the effect on threat detection metrics from benign nuisance sources of radiation,

5 such as medical isotopes, can be taken into account by the consuming anomaly detection or

6 source localization module, using nuisance-rejection as described in [1].

\section{$7 \quad 2.0$ Methodology}

8 We anticipate that aerial surveys will be conducted at altitudes typically ranging from $150 \mathrm{ft}$. to

$9300 \mathrm{ft}$. At these altitudes, the gamma-ray background radiation induced by cosmic rays should

10 be small compared to that from other sources, such as terrestrial radiation and radon. As a side

11 note, the cosmic-induced gamma-ray energy spectrum is expected to have an approximately $\mathrm{E}^{-1.3}$

12 energy dependence below a few $\mathrm{MeV}$, where $\mathrm{E}$ is the gamma-ray energy [7].

13 Terrestrial radiation is generated from primordial radionuclides in the earth's crust. These

14 radionuclides can be classified into three primary groups: $1{ }^{40} \mathrm{~K}(\mathrm{~K}), 2{ }^{235} \mathrm{U} /{ }^{238} \mathrm{U}$ and their

15 progeny $(\mathrm{U})$, and 3$)^{232} \mathrm{Th}$ and its progeny $(\mathrm{T})$. The radiation emitted from building materials,

16 including the aircraft itself, is also likely to be mostly generated from these three primary

17 radionuclide groups. The radon gamma-ray energy spectrum is structurally very similar to the $\mathrm{U}$

18 decay-series component, since its decay sequence contains ${ }^{214} \mathrm{~Pb}$ and ${ }^{214} \mathrm{Bi}$, which are the primary

19 gamma-ray emitters of the $\mathrm{U}$ decay series radiation component [8]; although, the lower-energy

20 photopeaks (e.g. $609 \mathrm{keV}$ ) are more pronounced in the radon contribution to the spectra,

21 particularly at higher altitudes, due to its proximity to the aircraft relative to the ground surface

$22[8]$. 
1 As an intermediate step to estimate the background spectrum, the current algorithm estimates the

$2 \mathrm{~K}, \mathrm{U}$, and $\mathrm{T}$ soil concentrations, namely $c_{\mathrm{K}}(\%), c_{\mathrm{U}}(\mathrm{ppm})$, and $c_{\mathrm{T}}(\mathrm{ppm})$, respectively, using

3 matrix equation (1), of the form $n_{3 \times 1}=S_{3 \times 3} c_{3 \times 1}$.

$$
\left(\begin{array}{c}
n_{\mathrm{K}} \\
n_{\mathrm{U}} \\
n_{\mathrm{T}}
\end{array}\right)=\left(\begin{array}{lll}
S_{\mathrm{K}, \mathrm{K}} & S_{\mathrm{K}, \mathrm{U}} & S_{\mathrm{K}, \mathrm{T}} \\
S_{\mathrm{U}, \mathrm{K}} & S_{\mathrm{U}, \mathrm{U}} & S_{\mathrm{U}, \mathrm{T}} \\
S_{\mathrm{T}, \mathrm{K}} & S_{\mathrm{T}, \mathrm{U}} & S_{\mathrm{T}, \mathrm{T}}
\end{array}\right)\left(\begin{array}{c}
c_{\mathrm{K}} \\
c_{\mathrm{U}} \\
c_{\mathrm{T}}
\end{array}\right)
$$

5 In this equation, the elements $n_{\mathrm{K}}, n_{\mathrm{U}}$, and $n_{\mathrm{T}}$ in vector $n$ are the counts measured over channels

6 corresponding to the gamma-ray energy windows defined in Table 1. These windows are

7 recommended by the IAEA for airborne gamma-ray spectroscopy measurements taken with

$8 \mathrm{NaI}(\mathrm{Tl})$ detectors [4]. The major contributing photopeaks to the standard $\mathrm{K}$, $\mathrm{U}$, and $\mathrm{T}$ windows

9 are also shown in Table 1 and in Fig. 1. A description is provided in section 2.2 of how the

10 NASVD algorithm is used, in real time, to obtain more accurate estimates of the true mean

11 values of $n_{\mathrm{K}}, n_{\mathrm{U}}$, and $n_{\mathrm{T}}$ and thus $c_{\mathrm{K}}, c_{\mathrm{U}}$, and $c_{\mathrm{T}}$. In equation (1), the sensitivity matrix $S$,

12 determined pre-flight, contains elements $s_{\mathrm{i}, \mathrm{j}}$. These are the counts measured in energy window $i$

13 from background component $j$, normalized by the concentration of $j$ in the soil. In this work, $S$

14 was determined using Monte Carlo radiation transport simulations, which are discussed in

15 section 2.1.

16 Table 1. Standard windows for determining K, U, and T concentrations [4]. The major

17 contributing photopeak to each window is shown.

\begin{tabular}{|c|c|c|}
\hline Window & Nuclide & Energy Range $(\mathrm{keV})$ \\
\hline $\mathrm{K}$ & ${ }^{40} \mathrm{~K}(1460 \mathrm{keV})$ & $1370-1570$ \\
\hline $\mathrm{U}$ & ${ }^{214} \mathrm{Bi}(1765 \mathrm{keV})$ & $1660-1860$ \\
\hline $\mathrm{T}$ & ${ }^{208} \mathrm{Tl}(2614 \mathrm{keV})$ & $2410-2810$ \\
\hline
\end{tabular}

In the current algorithm, since the primary objective is to simply estimate the background and not the actual $\mathrm{K}$, $\mathrm{U}$, and $\mathrm{T}$ concentrations in the soil, the cosmic and radon contributions to the

21 background are not explicitly calculated. Rather, the MLE-based calibration procedure

22 described later is relied upon to empirically fold these effects, as well as other extraneous effects,

23 such as atmospheric conditions, into the background estimates. 
2 In this work, the sensitivity matrix $S$ is determined as a function of altitude through the use of

3 Monte Carlo radiation transport simulations. Simulations using MCNP5 [9] were performed

4 with a model consisting of the helicopter above a $1 \times 1 \times 0.0015 \mathrm{~km}^{3}$ volume of soil and with an air

5 density of $1.199 \times 10^{-3} \mathrm{~g} / \mathrm{cm}^{3}$. The air was modeled with the following properties; a temperature

6 of $20^{\circ} \mathrm{C}$, a barometric pressure of 1013.25 mbar, and a relative humidity of $50 \%$. The helicopter

7 was modeled at altitudes ranging from $100 \mathrm{ft}$. to $900 \mathrm{ft}$. in $100 \mathrm{ft}$. increments. A surface source

8 was placed on top of the soil consisting of gamma rays from the $\mathrm{K}, \mathrm{U}$, and $\mathrm{T}$ background

9 components developed from a previous background modeling effort [7]. The individual K, U,

10 and T components of the detector response were tallied separately in the simulations, and the

11 Gaussian energy broadening feature was applied, as implemented in MCNP. The K, U, and T

12 spectra are shown in Fig. 1, using log-scale on the dependent axis, and offset for clarity. These

$13 \mathrm{~K}, \mathrm{U}$, and $\mathrm{T}$ detector response components were then normalized by the respective $\mathrm{K}$, $\mathrm{U}$, and $\mathrm{T}$

14 concentrations modeled in the soil, namely $412 \mathrm{~Bq}$ of $\mathrm{K}$ per $\mathrm{kg}$ of soil $(\mathrm{Bq} / \mathrm{kg}), 34 \mathrm{~Bq} / \mathrm{kg}$ for $\mathrm{U}$,

15 and $45 \mathrm{~Bq} / \mathrm{kg}$ for $\mathrm{T}$. Converting these units from $\mathrm{Bq} / \mathrm{kg}$ to per cent and parts per million [10],

16 these concentrations translate to $1.32 \% \mathrm{~K}, 2.75 \mathrm{ppm} \mathrm{U}$, and $11.1 \mathrm{ppm} \mathrm{T}$. Matrix elements $s_{\mathrm{K}, \mathrm{K}}$,

$17 s_{\mathrm{U}, \mathrm{U}}$, and $s_{\mathrm{T}, \mathrm{T}}$ of $S$ are shown in Fig. 2 as a function of the helicopter altitude, as modeled in the

18 simulation, above the homogenous soil volume. Exponential fits to these plots are also shown in

19 Fig. 2. From the simulations, the off-diagonal elements of $S$ also closely followed a decaying

20 exponential trend with altitude. Therefore, in the current background estimation algorithm, each

21 of the individual elements of $S$ are stored as exponential functions of height, which is read in

22 real-time from the aircraft's altimeter. 


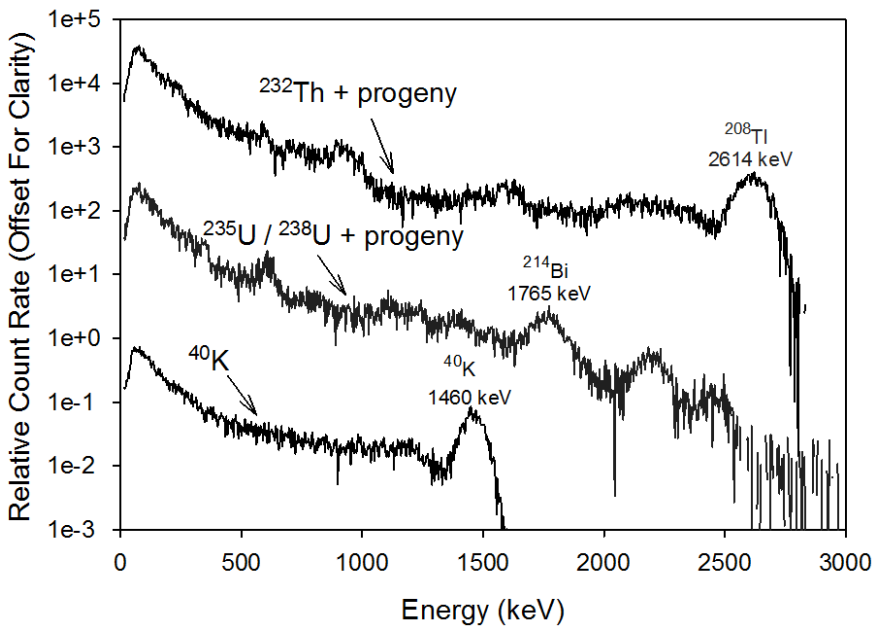

2 Fig. 1. Individual K, U, and T contributions to the response of the helicopter-mounted $\mathrm{NaI}(\mathrm{Tl})$

3 gamma-ray detectors, from the MCNP5 background simulations.

4

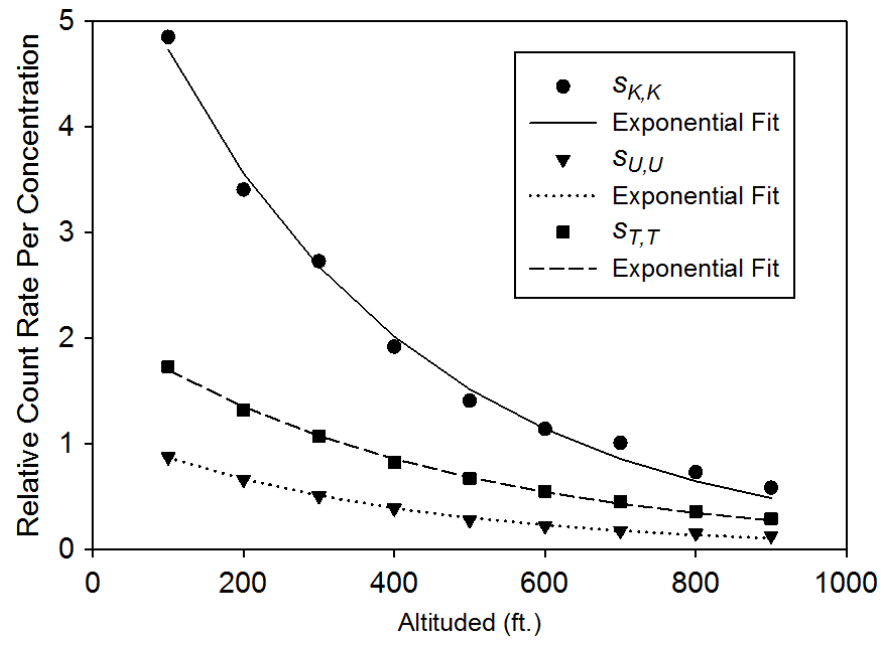

6 Fig. 2 Sensitivity matrix elements $s_{\mathrm{K}, \mathrm{K}}, s_{\mathrm{U}, \mathrm{U}}$, and $s_{\mathrm{T}, \mathrm{T}}$ as a function of altitude from the MCNP5

7 simulations. 
1 In geological surveying applications of airborne spectroscopy, measurements to determine the

2 sensitivity coefficients as a function of altitude (height) are typically taken over a test strip

3 (calibration range). The test strip should have, among several other properties, accessibility to

4 surface measurements and uniform concentrations of $\mathrm{K}, \mathrm{U}$, and $\mathrm{T}$ [4]. The attenuation

5 coefficients for each window are determined by fitting an exponential curve to a plot of the

6 stripped count rates (count rates from each component to its respective window) as a function of

7 the equivalent height, $H_{e}$, of the aircraft at a standard temperature and pressure (STP) of $0^{\circ} \mathrm{C}$ and

$8 \quad 1013.25$ mbar, respectively. $H_{e}$ is defined in equation (2) for measurements taken at an actual

9 height of $H$, a temperature of $T\left({ }^{\circ} \mathrm{C}\right)$, and a barometric pressure of $P$ (mbar) [4].

10

(2) $\quad H_{e}=H \times \frac{273.15}{T+273.15} \times \frac{P}{1013.25}$

11 In Table 2, attenuation coefficients, $\mu$, determined in this manner from previously measured

12 data are compared with the results obtained from the simulations in this work, adjusted to STP

13 using (2). The linear attenuation coefficients for the $\mathrm{K}, \mathrm{U}$, and $\mathrm{T}$ windows for the simulations

14 agree with data taken from previous measurements conducted by the IAEA [4] within 3\%, 5\%,

15 and $2 \%$, respectively.

16 Table 2. Linear attenuation coefficients calculated from exponential fits to the simulated results

17 compared with previous measurements.

\begin{tabular}{|c|c|c|}
\hline Window & $\begin{array}{c}\mu \text { from simulations } \\
\text { in current work } \\
\left(\mathrm{m}^{-1}\right)\end{array}$ & $\begin{array}{c}\mu \text { from previous } \\
\text { measurements } \\
\left(\mathrm{m}^{-1}\right)[4]\end{array}$ \\
\hline $\mathrm{K}$ & $9.17 \times 10^{-3}$ & $9.45 \times 10^{-3}$ \\
\hline $\mathrm{U}$ & $8.67 \times 10^{-3}$ & $8.28 \times 10^{-3}$ \\
\hline $\mathrm{T}$ & $7.72 \times 10^{-3}$ & $7.83 \times 10^{-3}$ \\
\hline
\end{tabular}

18 
2 The $\mathrm{K}, \mathrm{U}$, and $\mathrm{T}$ concentrations $\left(c_{\mathrm{K}}, c_{\mathrm{U}}\right.$, and $\left.c_{\mathrm{T}}\right)$ are estimated in real time from the data taken

3 during flight. It is very difficult to obtain even moderately precise estimates of the $c_{\mathrm{K}}, c_{\mathrm{U}}$, and $c_{\mathrm{T}}$

4 from raw (un-processed) one-second airborne spectra due to the significant inherent noise from

5 poor counting statistics. Therefore, noise-adjusted singular value decomposition (NASVD) [11]

6 was used in real time to smooth the measured one-second spectra to improve the $c_{\mathrm{K}}, c_{\mathrm{U}}$, and $c_{\mathrm{T}}$

7 estimates. For large airborne gamma-ray spectrometric surveys, the NASVD typically reduces

8 the fractional errors in $c_{\mathrm{K}}, c_{\mathrm{U}}$, and $c_{\mathrm{T}}$ by $14 \%, 42 \%$, and $37 \%$, respectively, relative to extracting

9 these concentrations directly from the raw spectra [12]. The NASVD is applied by scaling the

10 measured, observed spectra so that they have unit variance in each of their channels,

11 accomplished as follows [12]. Prior to the SVD operation, each of the observed spectra is

12 divided, channel by channel, by the least-squares fit of the average spectrum to the respective

13 observed spectrum. A matrix is formed from these scaled (noise-adjusted) spectra, and the SVD

14 is applied to this matrix. The resulting principle components from the SVD are then multiplied,

15 channel by channel, by the average spectrum to reverse the scaling. The noise-adjustment, in

16 effect, flattens the observed spectra and thus reduces the variation that the principle components

17 from the SVD operation must span.

18 To implement the NASVD in real time, a dynamic buffer of the past 15 one-second raw spectra, 19 each containing 1024 channels, was stored in a $15 \times 1024$ matrix. At each second in flight, the 20 most recently-measured spectrum was placed into this matrix along with the previously21 measured 14 raw spectra. Traditionally [11-12], the resulting smoothed spectra are obtained

22 from the NASVD by multiplying the unit PCs (eigenvectors) by the sum of the counts in each

23 raw, respective spectrum prior to reconstruction. In this work, however, slightly, yet 
1 consistently more accurate background estimates were obtained by forming a smoothed version,

$2 x$, of the recently-measured raw spectrum according to equation (3). In (3), $v_{1}, v_{2}$, and $v_{3}$ are first

3 three, lower-order principle components (PCs) obtained by applying the NASVD over the

4 dynamic buffer matrix. Coefficients $r, s$, and $t$ in (3) were determined by fitting $x$ to the recently-

5 measured raw spectrum using ordinary least squares (OLS).

$6 \quad(3) \quad x=r v_{1}+s v_{2}+t v_{3}$

7 More robust fitting techniques and the use of greater than three PCs have been employed but

8 have not appreciably improved the background estimates on the examined data sets.

9 The counts, $n_{\mathrm{K}}, n_{\mathrm{U}}$, and $n_{\mathrm{T}}$ are obtained from the $\mathrm{K}, \mathrm{U}$, and T energy windows in the NASVD-

10 smoothed spectrum. Using $S$, matrix equation (1) is then solved for concentration vector $c$,

11 containing $c_{\mathrm{K}}, c_{\mathrm{U}}$, and $c_{\mathrm{T}}$. Results are shown in Table 3 for the average $c_{\mathrm{K}}, c_{\mathrm{U}}$, and $c_{\mathrm{T}}$ estimates

12 using the current algorithm, at a nominal flying altitude of $100 \mathrm{ft}$., over a fairly homogenous

13 region of the Government Wash 7.5-Minute Quadrangle near Las Vegas, Nevada. These results

14 are compared to interpolated values over United States Geological Survey (USGS) Maps [13]. It

15 should be noted that the USGS maps are fairly sparse in this region, but can serve as a rough

16 estimate. The agreement is generally good between the estimated $c_{\mathrm{K}}, c_{\mathrm{U}}$, and $c_{\mathrm{T}}$ values. The

17 larger discrepancy in the values for $c_{\mathrm{T}}$ is likely due to the low level of counts within the high-

18 energy $\mathrm{T}$ window, for which the detection efficiency is lower.

19 Table 3. Comparison of $c_{\mathrm{K}}, c_{\mathrm{U}}$, and $c_{\mathrm{T}}$ estimates

\begin{tabular}{|c|c|c|}
\hline concentrations & This work (100 ft.) & Interpolated USGS [13] \\
\hline$c_{\mathrm{K}}(\%)$ & 0.57 & 0.51 \\
\hline$c_{\mathrm{U}}(\mathrm{ppm})$ & 3.11 & 3.27 \\
\hline$c_{\mathrm{T}}(\mathrm{ppm})$ & 3.09 & 2.56 \\
\hline
\end{tabular}


2 One method by which the background contribution in each channel of the measured spectrum

3 could be estimated during flight is by determining the $c_{\mathrm{K}}, c_{\mathrm{U}}$, and $c_{\mathrm{T}}$ using the method described

4 in section 2.2 and then using these concentrations to scale the $\mathrm{K}, \mathrm{U}$, and $\mathrm{T}$ spectra from the

5 simulations. This method provided good estimates for higher regions of the background

6 spectrum, but overshot the actual background in the lower energy regions, less than

7 approximately $80 \mathrm{keV}$. A graph comparing the $30 \mathrm{~min}$. average spectrum measured over the

8 homogeneous region of Government Wash is compared with the MCNP-simulated spectrum in

9 Fig. 3. The simulated spectrum has been scaled to the K, U, and $\mathrm{T}$ concentrations estimated in

10 this work, listed in Table 3, for this geographical area. The discrepancy between the measured

11 and simulated spectra is likely a result of the detector electronics effects below approximately 40

$12 \mathrm{keV}$ (measurements using $\mathrm{NaI}$ often set the lower discriminator close to $40 \mathrm{keV}$ ) and lack of

13 sufficient detail in the model, such as attenuating materials, up to $80 \mathrm{keV}$. Over-estimations of

14 the $\mathrm{K}, \mathrm{U}$, and $\mathrm{T}$ concentrations made these low-energy discrepancies more apparent. Also, due

15 to the assumptions made in the MCNP5 simulations, estimating the background in this way

16 would likely require cosmic and radon corrections, particularly at the higher search altitudes. 


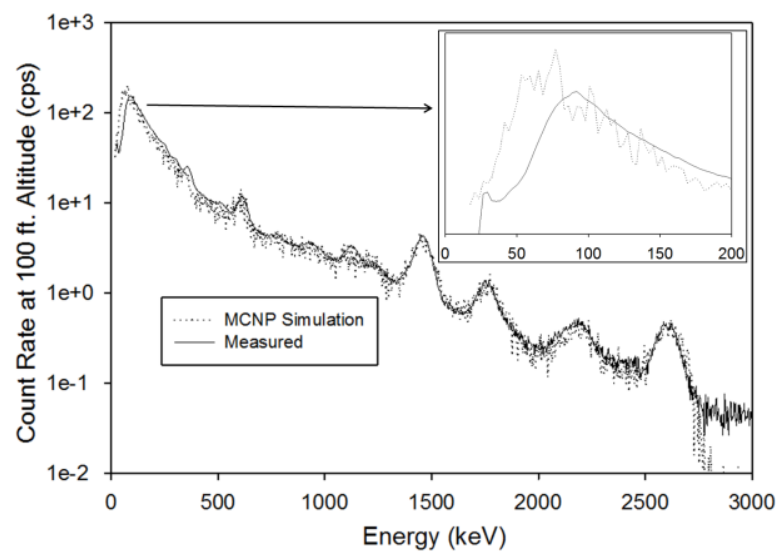

2 Fig. 3 Comparison between measured and MCNP-simulated spectra over the homogenous region 3 of Government Wash.

5 A Poisson regression technique was developed to produce a set of basis vectors using spectra 6 measured during previous flights. This technique is based on the assumption that the counts in

7 each channel of the background spectrum are Poisson-distributed and can be modeled as a linear 8 combination of the individual $\mathrm{K}, \mathrm{U}$, and $\mathrm{T}$ spectra as in (4). In (4), $y_{i}$ is simply the estimate, 9 produced by the Poisson regression technique, of the background portion of counts in the $i$-th 10 channel of a spectrum.

$$
y_{i}=a_{i} c_{\mathrm{K}}+b_{i} c_{\mathrm{U}}+c_{i} c_{\mathrm{T}}
$$

12 In (4), $a_{i}, b_{i}$, and $c_{i}$ are unknown scaling coefficients corresponding to the $i$-th channel and are 13 determined through optimization by the Poisson regression. Using this mathematical model, a 14 method based on maximum-likelihood estimation (MLE) can be applied to a calibration set of 15 data taken over a previous flight, ideally under similar conditions. This is described 16 mathematically by (5). 


$$
f_{i}=\sum_{j=1}^{N}\left(y_{i, j}-Y_{i, j} \log y_{i, j}\right)
$$

2 In (5), the subscript $j$ is added to show that $y_{i, j}$, and thus constants $a_{i}, b_{i}$, and $c_{i}$ through relation

3 (4), are optimized over $N$ calibration spectra, each denoted by $j$. The term $Y_{i, j}$ is the actual,

4 observed number of counts in calibration spectrum $j$, and $f_{i}$ is the so-called objective function for

5 channel $i$. This objective function is found by taking the negative-log of the Poisson distribution

6 function in parameter $Y_{i, j}$ with assumed distribution mean $y_{i, j}$. This objective function, $f_{i}$, is then

7 minimized by finding the optimum values of $y_{i, j}$, and thus parameters $a_{i}, b_{i}$, and $c_{i}$. It is noted

8 that minimizing the negative-log likelihood is equivalent to maximizing the log-likelihood. In

9 implementing this approach, the Newton-Raphson method was used, and OLS was used to

10 generate an initial estimate for this iterative procedure. An MLE calibration using 1000 spectra,

11 obtained from slightly less than 17 minutes of flight time, was found to be sufficient and required

12 less than three seconds to perform using MATLAB [14] on a desktop computer having a 2.3

$13 \mathrm{GHz}$ processor. Therefore, more sophisticated quasi-Newton algorithms, although more efficient

14 than the Newton-Raphson method, were not investigated to minimize (5).

15 The result of this MLE-based calibration procedure is a $1024 \times 3$ scaling matrix containing the $a_{i}$,

$16 b_{i}$, and $c_{i}$ 's for each of the 1024 channels in the spectrum. This method was used to produce

17 scaling matrices at $100 \mathrm{ft}$., $200 \mathrm{ft}$., and $400 \mathrm{ft}$. flight altitudes over the heterogeneous region of

18 Government Wash. During background estimation, linear interpolation over flight altitude, read

19 from the altimeter, was used to calculate the scaling matrix. To estimate the background, as each

20 raw spectrum is measured during flight, this matrix can be multiplied by the vector $c$ containing

$21 c_{\mathrm{K}}, c_{\mathrm{U}}$, and $c_{\mathrm{T}}$ determined using the method described in section 2.2. It should be noted that this 
matrix is also altitude-dependent, for which the $b_{i}$ 's, which can also be viewed as the

2 empirically-determined $U$ basis vector, is shown in Fig. 4.

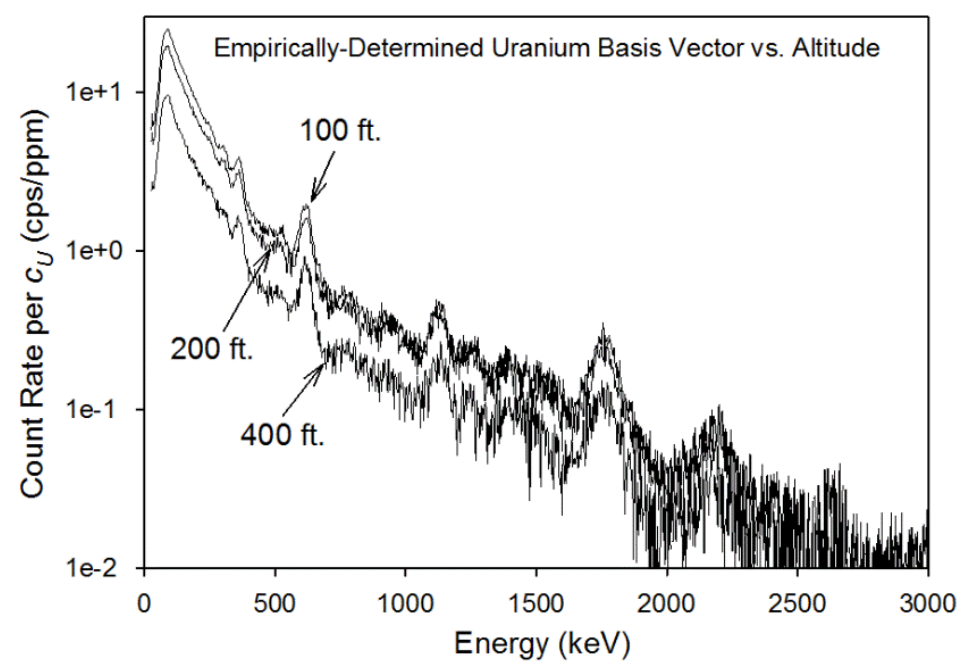

4 Fig. 4 The U basis vector, determined using the MLE-based calibration procedure, as a function 5 of flight altitude.

\section{$6 \quad 3.0$ Results}

7 Results are shown in Fig. 5, for which a ${ }^{60}$ Co source was placed in the homogenous region of

8 Government Wash using scaling matrices calculated by performing the MLE-based calibration

9 method described in section 2.3. The nominal flying altitude was $100 \mathrm{ft}$. The spectrum labelled ${ }^{60} \mathrm{Co}$ and the estimated background spectrum are for a one-second snapshot during the flight at

11 which the ${ }^{60} \mathrm{Co}$ source generated the largest number of counts in the gamma-ray detectors. This

12 number was slightly over twice the gross count rate of the average background, which is also

13 shown in Fig. 5. This average background was taken over the same homogenous region during a

14 previous flight with no source present other than background and therefore is considered ground

15 truth for this scenario. These results are encouraging in that the current algorithm produced a

16 smoothed, accurate estimate of the background and was not noticeably affected by the strong, 
1 fairly high-energy ${ }^{60} \mathrm{Co}$ source. In the observed one-second spectrum, the counts within the $\mathrm{K}$,

$2 \mathrm{U}$, and T energy regions, listed in Table 1 , were 224,44 , and 21, respectively.

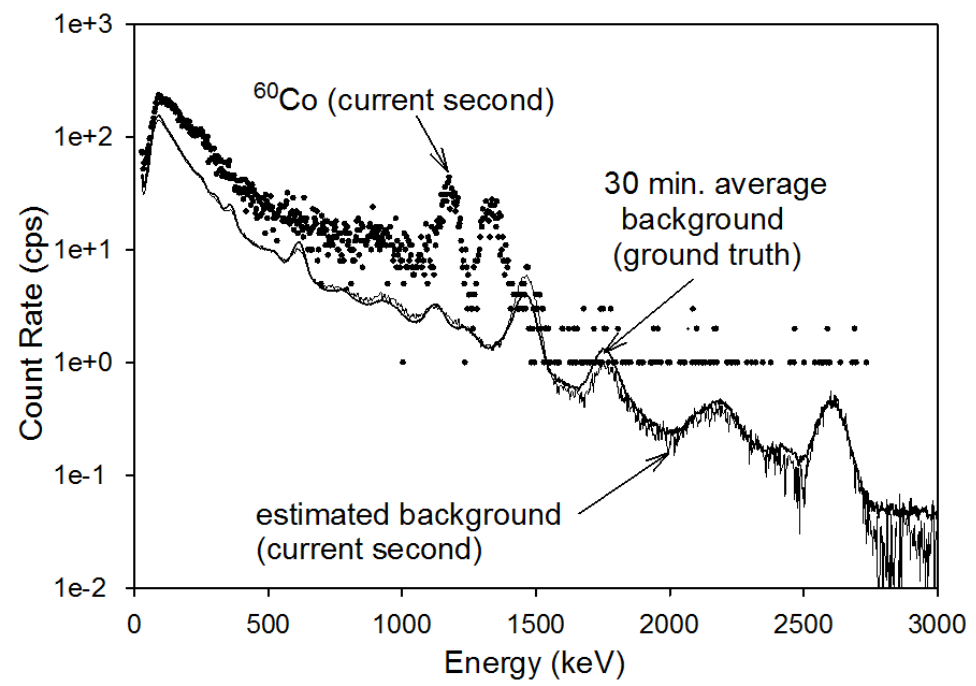

4 Fig. 5 Background spectrum estimate over a homogenous region of Government Wash with ${ }^{60} \mathrm{Co}$ 5 source present.

6 The three PCs from the NASVD, which were used to fit the one-second ${ }^{60}$ Co spectrum shown in

7 Fig. 5, are given in Fig. 6 as a function of energy. In the Fig. 6 inset, the first two PCs are given

8 between $1400 \mathrm{keV}$ and $2800 \mathrm{keV}$ to illustrate the primary photopeaks, listed in Table 1, in each

9 of the background components. The third PC consisted entirely of noise at these high energies

10 and is therefore, for the purpose of clarity, not shown in the Fig. 6 inset. With (3), a linear

11 combination of these three PCs was used to fit, and to the extent possible, project unwanted noise

12 out of the one-second ${ }^{60} \mathrm{Co}$ spectrum in order to reduce the uncertainty of the total counts within

13 the $\mathrm{K}, \mathrm{U}$, and $\mathrm{T}$ energy windows. Equation (1) was then used to determine the $\mathrm{K}, \mathrm{U}$, and $\mathrm{T}$

14 concentrations from the counts within these three windows in the spectral fit. The three basis

15 vectors, determined from the MLE calibration procedure described in section 2.3, were then 
1 scaled by these calculated $\mathrm{K}, \mathrm{U}$, and $\mathrm{T}$ concentrations to generate the final background estimate 2 shown in Fig. 5.

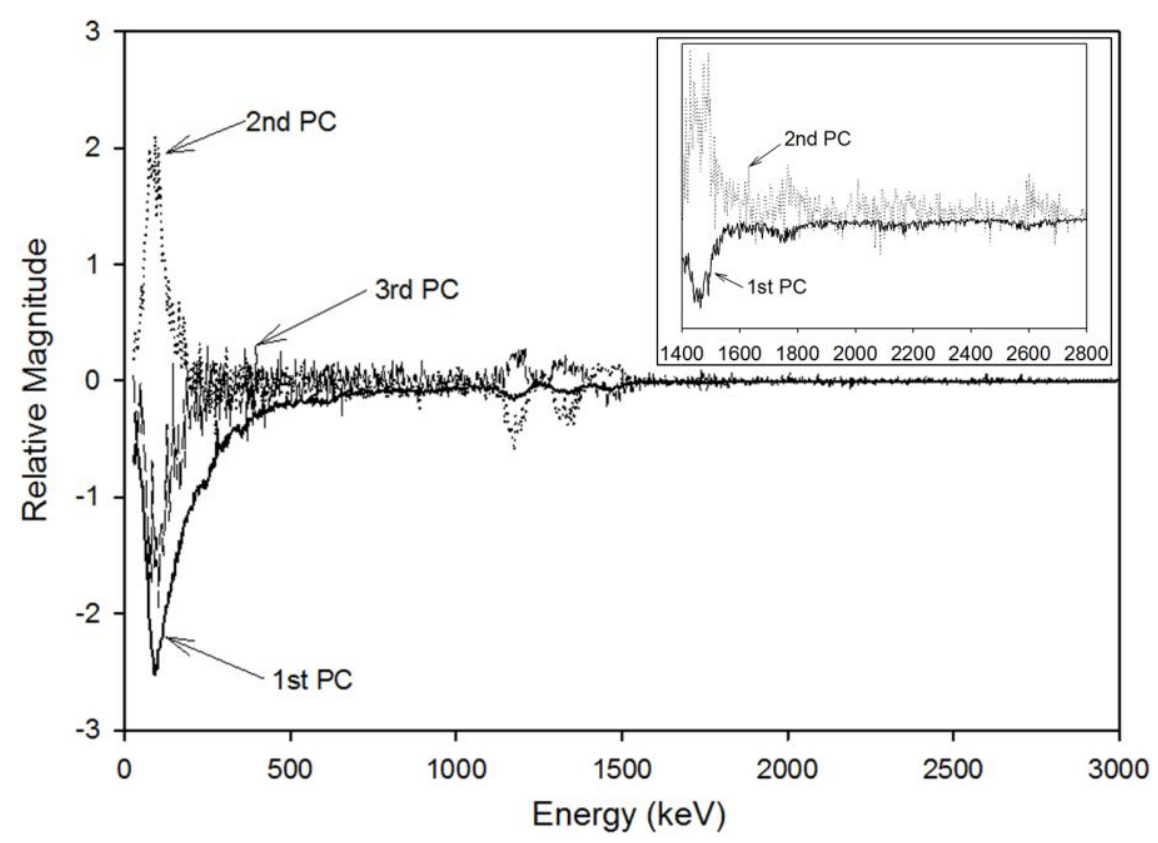

4 Fig. 6 The three PCs from NASVD used to fit the one-second ${ }^{60}$ Co spectrum shown in Fig. 5.

6 The same calibration data over the complex, heterogeneous region of Government Wash was

7 used to estimate the background during a $\sim 2$ hour flight over an urban area containing both

8 Oakland, CA and Berkeley, CA. The flight was conducted at an altitude of $300 \mathrm{ft}$. The results

9 are shown in Fig. 7 for the worst (largest) and typical (median) chi-square fit to the

10 corresponding measured, one-second spectra. The worst-fit spectrum was taken over the San

11 Francisco Bay and had a count rate approximately 10 times lower than the spectrum with the

12 typical fit. This spectrum has been divided by a factor of 10 in Fig. 7 for clarity. No ground

13 truth background estimates were available for these background estimates; although, the results

14 are quite encouraging in that estimates agree fairly well with the low-energy regions of the 
1 measured spectra using calibration data over a very different region. In MATLAB, a single

2 background estimate required an average of 0.0267 seconds on a computer with a $2.3 \mathrm{GHz}$

3 processor.

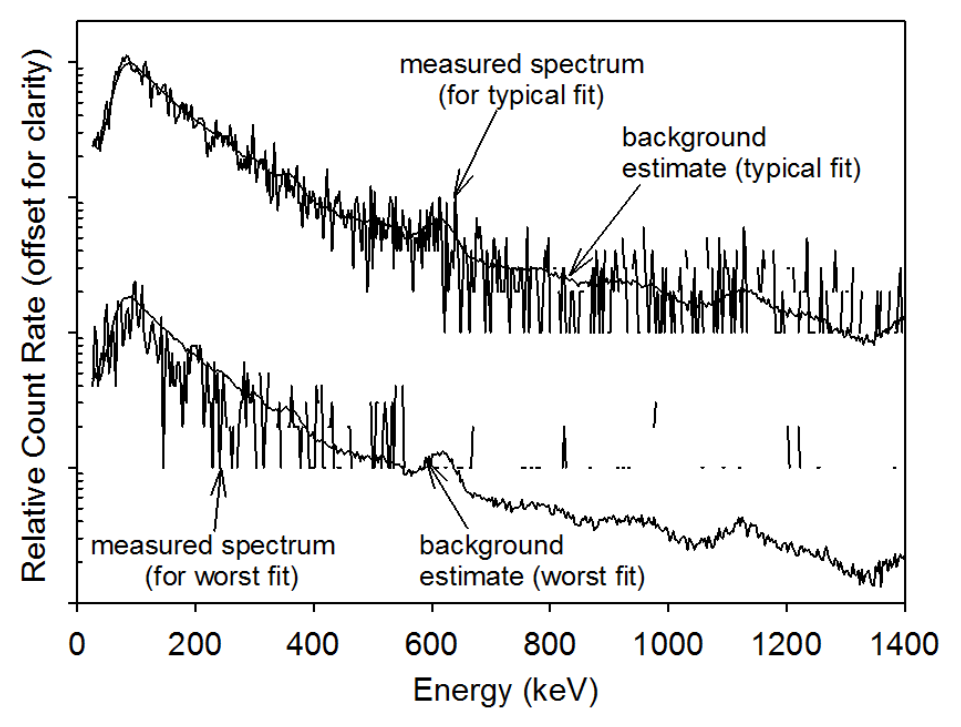

5 Fig. 7 Background estimates at $300 \mathrm{ft}$. altitude over an urban area containing both Berkeley, CA 6 and Oakland, CA.

8 One of the more challenging data sets that was investigated was one for which the helicopter

9 flew several times over a land-water interface. The background count rate changes significantly

10 when traversing over this interface, since the background measured over water is almost entirely

11 from radon and cosmic components. These results are shown in terms of gross counts for

12 representative paths of land to water in Fig. 8 and in Fig. 9 for water to land flight paths. As

13 shown, when flying from land to water, there is an approximately 15 -second lag in the algorithm.

14 The discrepancy was not as great when flying from water to land, but the reason for this is

15 unknown. A smaller dynamic buffer of 10 rather than 15 spectra was applied, but did not appear 
1 to significantly improve the agreement between the estimated and measured background, as

2 illustrated in Fig. 8 and in Fig. 9.

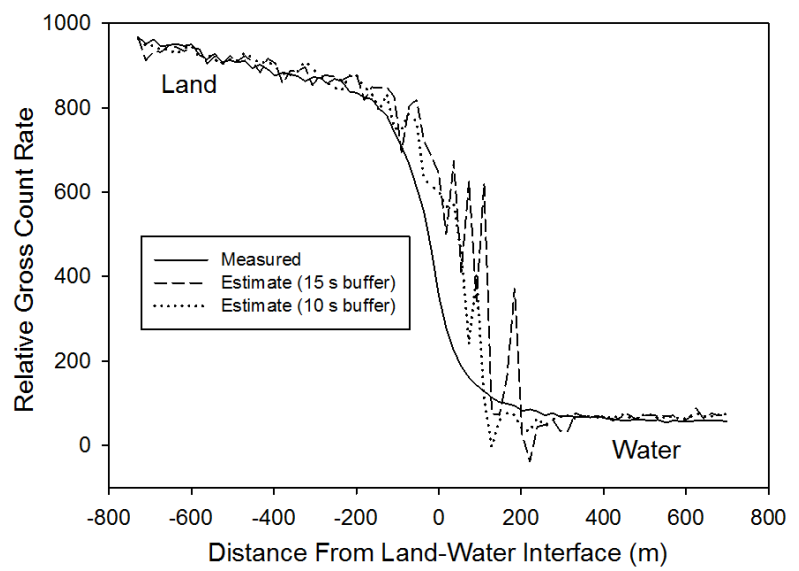

4 Fig. 8 Estimated vs. measured count rate for flight path from land to water at an altitude of 100 $5 \mathrm{ft}$.

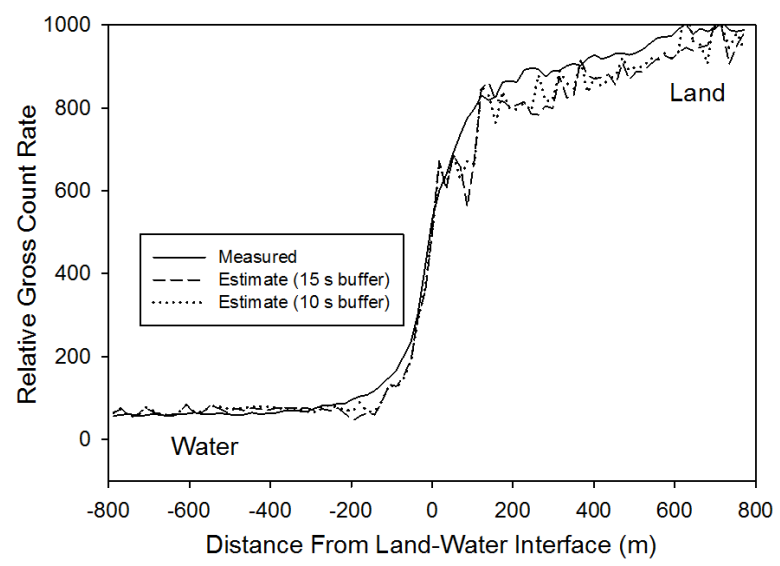

8 Fig. 9 Estimated vs. measured gross count rate for flight path from water to land at an altitude of $9100 \mathrm{ft}$.

10 A simulated scenario was also investigated, in which a step change of $c_{\mathrm{K}}$ and $c_{\mathrm{T}}$ were inserted 11 into the simulation. The purpose of this exercise was to determine how well the dynamic buffer 
1 with NASVD could adjust to large and abrupt changes in background. The K, U, and T

2 empirical basis vectors from the homogenous region of Government Wash were extracted from

3 the actual flight spectra, measured at an altitude of $100 \mathrm{ft}$, using the MLE method described in

4 section 2.2. The scenario was developed by scaling these basis vectors by the calculated $c_{\mathrm{K}}, c_{\mathrm{U}}$,

5 and $c_{\mathrm{T}}$ values shown in Table 3, until the step change. At a point precisely in the middle of the

6 flight, $c_{\mathrm{K}}$ was multiplied by $10, c_{\mathrm{T}}$ was divided by 10 , and $c_{\mathrm{U}}$ was left unchanged. Poisson noise

7 was added to simulate the noise in the spectra. This may represent a somewhat unrealistic

8 scenario for which the ${ }^{40} \mathrm{~K}$ and ${ }^{232} \mathrm{Th}$ background components suddenly change beneath the

9 helicopter. The results are shown in Fig. 10, which illustrates the spectrum that the helicopter

10 would theoretically measure immediately prior to the step change, the actual background at the

11 instant of the step change, and the estimated background signature. As one may expect, the

12 estimated background significantly underestimates the ${ }^{40} \mathrm{~K}$ peak; although, the background

13 estimate is much closer in agreement at the lower energies of interest for radiological search.

14 The same calibration matrix from the Government Wash complex region was used for this

15 scenario to calculate the background estimates. 


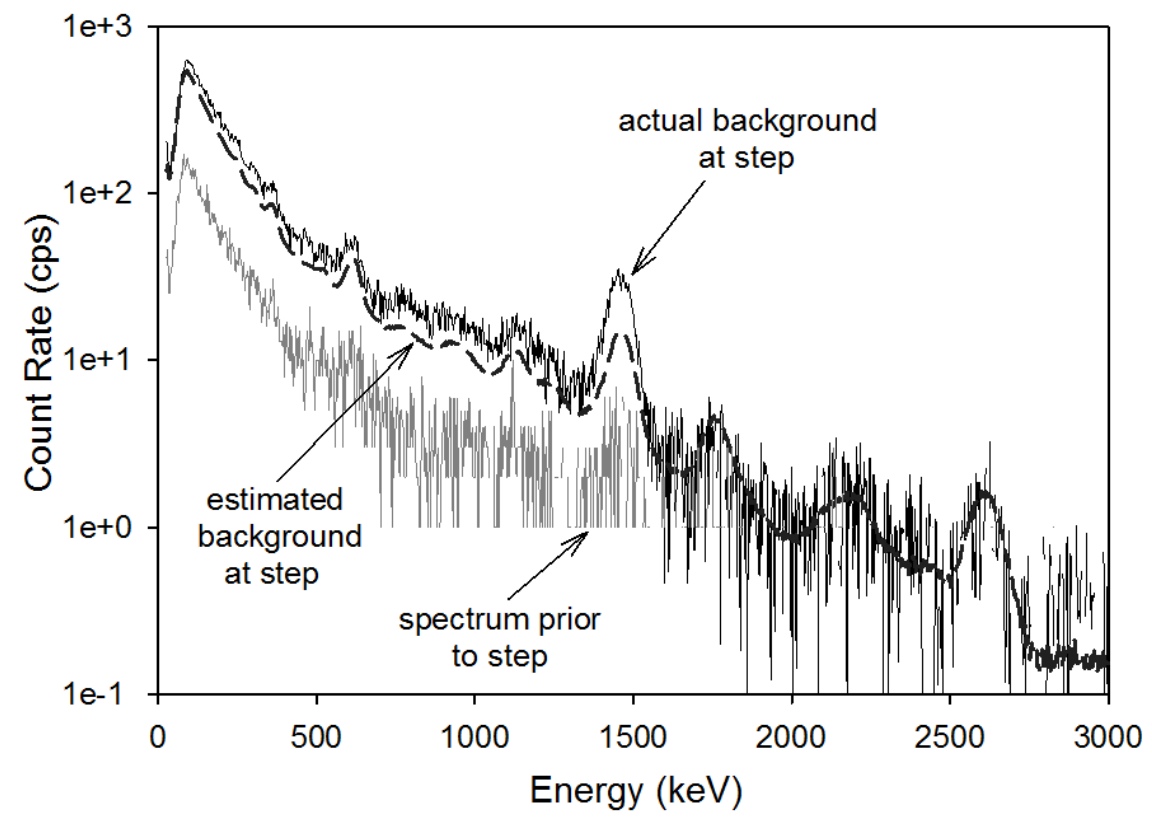

2 Fig. 10. Results of a simulated scenario for which a large step change in $c_{\mathrm{K}}$ and $c_{\mathrm{T}}$ were inserted 3 into the simulated background.

\subsection{Conclusions}

6 A new technique for real-time estimation of background gamma radiation from aerial

7 measurements for radiological search has been developed using a combination of Monte Carlo

8 calculations, NASVD, and MLE for calibration. It has been demonstrated to be accurate and

9 robust against contamination even in the presence of a strong ${ }^{60}$ Co source. The algorithm

10 produced accurate background estimates for rural and urban environments using calibration data

11 from a flight over a very different area. Results from a simulated scenario indicate that the

12 algorithm has good potential to track large and abrupt changes in background spectral shape and

13 magnitude. In MATLAB the average time to produce a background estimate was 0.0267

14 seconds on a computer with a $2.3 \mathrm{GHz}$ processor. The algorithm can be implemented fairly 
1 easily using the built-in mathematical libraries and functions available in most modern

2 computing languages and environments.

3 Several concepts can be explored to improve the accuracy of the background estimations. For

4 example, the sensitivity matrix used for extracting $c_{\mathrm{K}}, c_{\mathrm{U}}$, and $c_{\mathrm{T}}$ from the measured spectra in

5 this work was determined using Monte Carlo simulations, with the helicopter modeled parallel to

6 the soil. Currently, simulations are being performed to take into account several angular

7 orientations of the helicopter. Incorporating the results of these simulations to determine the

8 sensitivity matrix may improve the background estimates, for instance, when the helicopter is

9 turning during flight, which would likely skew the measured background spectra toward lower

10 energies. Incorporation of radon and cosmic effects may also potentially improve the validity of

11 (3) and thus the background estimates. Good detector gain stabilization is required for this

12 algorithm, since it relies heavily on calibrated data. Also, since the Gaussian energy broadening

13 feature as implemented in MCNP was used in this work to determine $S$, the resolution of the

14 detection system must be known prior to and remain fairly constant across the calibration and 15 survey flights.

\subsection{Acknowledgements}

17 This work has been supported by the US Department of Homeland Security, Domestic Nuclear

18 Detection Office, under competitively awarded contract/IAA HSHQDC-12-X-00376. This

19 support does not constitute an express or implied endorsement on the part of the Government.

20 This report is PNNL-SA-103906. Pacific Northwest National Laboratory is operated for the U.S.

21 Department of Energy by Battelle under Contract DE-AC05-76RL01830. The authors wish to

22 acknowledge helpful discussions with Dr. Kevin K. Anderson. 
2 [1] D. M. Pfund, R. C. Runkle, K. K. Anderson, and K. D. Jarman, "Examination of Count-

3 Starved Gamma Spectra Using the Method of Spectral Comparsion Ratios," IEEE Trans. Nucl.

$4 \quad$ Sci., vol. 54, no. 4, pp. 1232-1238, Aug. 2007.

5 [2] K. D. Jarman, E. A. Miller, R. S. Wittman, and C. J. Gesh, "Bayesian Radiation Source

6 Localization." Nucl. Technol., vol. 175, No. 1, pp. 126-334, 2011.

7 [3] M. Alamaniotis, J. Mattingly, and L. H. Tsoukalas, "Kernel-Based Machine Learning for

8 Background Estimation of NaI Low-Count Gamma-Ray Spectra," IEEE Trans. Nucl. Sci., vol.

9 60, No. 3, pp. 2209-2221, 2013.

10 [4] “Airborne Gamma-Ray Spectrometer Surveying," International Atomic Energy Agency,

11 Technical Report Series, No. 232, 1991.

12 [5] H. K. Aage and U. Korsbech, "Search for Ophan Radioactive Sources Based on NaI 13 Gamma Spectrometry," Appl. Radiat. and Isot., vol. 58, pp. 103-113, 2003.

14 [6] H. K. Aage, et al, "Experiences with Area Specific Spectrum Stripping of NaI(Tl)

15 Gamma Spectra," Radiat. Prot. Dosim., vol. 121, No. 2, pp. 108-121, 2006.

16

[7] G. A. Sandness, J. E. Schweppe, W. K. Hensley, J. D. Borgardt, and A. L. Mitchell,

17 "Accurate Modeling of the Terrestrial Gamma-Ray Background for Homeland Security

18 Applications," IEEE Nucl. Sci. Conf. R. (NSS/MIC), vol. N06-5, pp. 126-133, 2009.

19 [8] B. R. S. Minty, "Multichannel Models for the Estimation of Radon Background in

20 Airborne Gamma-Ray Spectrometry," Geophysics, vol. 63, No. 6, pp. 1986-1996, 1998. 
1 [9] MCNP X-5 Monte Carlo Team, "MCNP - A General Purpose Monte Carlo N-Particle

2 Transport Code, Version 5," LA-UR-03-1987, Los Alamos National Laboratory, Los Alamos,

3 NM, 2003.

4 [10] IAEA, "Construction and Use of Calibration Facilities for Radiometric Field Equipment,"

5 Technical Report 309, Vienna: International Atomic Energy Agency, 1989.

6 [11] J. Hovgaard, 1997. “A New Processing Technique for Airborne Gamma-Ray

7 Spectrometer Data (Noise Adjusted Singular Value Decomposition),” Am. Nucl. Soc. Sixth

8 Topical Meeting on Emergency Preparedness and Response, pp. 123-127, San Francisco, April

$9 \quad 22-25,1997$.

10 [12] B. Minty and P. McFadden, "Improved NASVD Smoothing of Airborne Gamma-Ray

11 Spectra," Explor. Geophys., vol. 29, pp. 516-523, 1998.

12 [13] J. S. Duval, J.M. Carson, P.B. Holman, and A.G. Darnley, "Terrestrial Radioactivity and 13 Gamma-Ray Exposure in the United States and Canada." U.S. Geological Survey, Washington, 14 D.C, 2005, Revised 2009.

15 [14] MATLAB version 7.14.0.739. Natick, Massachusetts: The MathWorks Inc., 2012. 